\title{
Recall of common and distinctive features of verbal and pictorial stimuli
}

\author{
ITAMAR GATI \\ Hebrew University, Jerusalem, Israel \\ and \\ AMOS TVERSKY \\ Stanford University, Stanford, Califormia
}

\begin{abstract}
Subjects first rated similarity of verbal and of pictorial stimuli. The same pairs of stimuli were presented again, without one common component and one distinctive component. Subjects were asked to recall the missing components and identify them as common or distinctive. The results show that components encoded as common are recalled relatively better than components encoded as distinctive, and the difference in recall is significantly greater in verbal than in pictorial stimuli.
\end{abstract}

Many tasks involve comparisons between objects, such as pictures, faces, letters, or words. Such comparisons induce a partition of the relevant information into features shared by the two objects and features that distinguish between them. The relative salience of the common and the distinctive features is an important determinant of the proximity of objects as measured, for example, by ratings of similarity, free classification, and recognition errors. Indeed, in the contrast model (Tversky, 1977), the similarity between objects is a linear function of the measures of their common and distinctive features. Naturally, similarity increases with the measure of the common features and decreases with the measure of the distinctive features.

The contrast model can be used to assess the relative weight of common and distinctive features. Let $s(p, q)$ denote the similarity between two objects $p$ and $q$ (e.g., schematic faces) and let $x$ denote an independent additive component (e.g., glasses). Adding $x$ to both $p$ and $q$ increases similarity, and this increment provides an estimate of the impact of $x$ as a common feature, denoted $C(x)$. That is,

$$
C(x)=s(p x, q x)-s(p, q) .
$$

Adding $x$ to only one object reduces similarity, and this decrement provides an estimate of the impact of $x$ as a distinctive feature, denoted $D(x)$. That is,

$$
D(x)=s(p, q)-s(p x, q)
$$

Gati and Tversky (1984) applied this method to many sets of verbal stimuli (e.g., descriptions of persons, meals,

This research was supported by a grant from the United States-Israe Binational Science Foundation (BSF), Jerusalem, Israel. Please address reprint requests to: Amos Tversky, Department of Psychology, Building 420, Stanford University, Stanford, CA 94305. trips) and of pictorial stimuli (e.g., schematic faces, landscapes) and estimated $C$ and $D$ for more than 50 components. These estimates revealed a highly consistent pattern: $C$ was greater than $D$ for almost all the verbal components, whereas $D$ was greater than $C$ for almost all the pictorial components.

The interaction between stimulus modality (verbal vs. pictorial) and feature type (common vs. distinctive) was observed in ratings of similarity and dissimilarity. The present study investigates whether this interaction is limited to judgment or whether it extends to memory. In particular, we test the hypothesis that components that were encoded as common are recalled relatively better in verbal than in pictorial stimuli, whereas components that were encoded as distinctive are recalled relatively better in pictorial than in verbal stimuli.

\section{METHOD}

\section{Stimuli}

Ten sets of verbal stimuli and $\mathbf{1 0}$ sets of pictorial stimuli were employed. Each stimulus set included two substitutive components (labeled $p$ and $q$ ) and two additive components (labeled $x$ and $y$ ). Thus, each stimulus included one of the substitutive components and one or two of the additive components. For each set, two pairs of stimuli $(p x, q x y)$ and $(p x y, q y)$ were constructed, so that each pair had one common and one distinctive additive component. The verbal and the pictorial stimulus sets are presented in Tables 1 and 2 , respectively. Three examples of pairs of pictorial stimuli are displayed in Figure 1. The pictorial stimuli were selected so that the additive components (e.g., cloud, tree, glasses) are physically distinct and easily named.

\section{Procedure}

One hundred twenty undergraduates from the Hebrew University in Jerusalem participated in the experiment, which consisted of two phases: comparison and recall.

In the first phase each subject was presented with 10 pairs of pictorial stimuli and 10 pairs of verbal stimuli. The stimuli were 
Table 1

Verbal Stimuli

\begin{tabular}{|c|c|c|c|c|}
\hline \multirow[b]{2}{*}{ Stimulus Set } & \multicolumn{2}{|c|}{ Substitutive Components } & \multicolumn{2}{|c|}{ Additive Components } \\
\hline & $p$ & $\underline{q}$ & $x$ & $y$ \\
\hline Symptoms & Fever & Weakness & Nausea & Sideache \\
\hline College Students & Economics Major & Biology Major & Pacifist & Introvert \\
\hline Professionals & Politician & University Professor & Religious & Persuasive \\
\hline High School Students & Science Major & Humanities Major & Red Hair & Scout Leader \\
\hline Art School Programs & Painting & Sculpture & Graphics & Product Design \\
\hline Trips (Israel) & Old City (Jerusalem) & Safari (Ramat-Gan) & Golan Heights & Eilat \\
\hline Trips (Europe) & Italy & France & Austria & Switzerland \\
\hline Trips (USA) & New York & Washington & Boston & Miami \\
\hline Breakfasts & Cereal & Omelet & Toast & Juice \\
\hline Dinners & Schnitzel & Steak & Vegetable Soup & Watermelon \\
\hline
\end{tabular}

presented in booklets. The pictorial stimuli were presented side by side, as in Figure 1, but without the labels. The verbal stimuli (e.g., descriptions of students) were presented as follows:

- Biology major, pacifist, introvert

- Economics major, introvert

To encourage the subject to compare the elements of each pair and to note their common and distinctive components, we asked subjects to rate the similarity of each pair on a 6-point scale. One half of the subjects rated the pairs ( $p y, q x y)$, and the other half rated the pairs $(p x y, q x)$. Each additive component, therefore, appeared both as a common and as a distinctive feature. In half of the questionnaires, the verbal pairs appeared before the pictorial ones, and in the other half the order was reversed.

In the second phase, the same pairs of stimuli were presented (in a different random order), but the additive components $x$ and $y$ were deleted. The instructions read as follows:

In this part of the questionnaire you will be presented with the same pairs of items, but without some components. You are asked to recall the two missing (deleted) components and to write them down. Note that one of the deleted components was included in both items, and the other was included in one item only.

For both verbal and pictorial stimuli, the subjects were asked to report, in words, the missing components. For the above pair of descriptions of students, for instance, the questions read as follows:

- Biology major

- Economics major

What is the (missing) component that appeared in both items? What is the (missing) component that appeared in one item only?

Half of the subjects were asked to recall first the missing common component and then the missing distinctive component. The order was reversed for the other half.

Table 2

Pictorial Stimuli

\begin{tabular}{|c|c|c|c|c|}
\hline \multirow[b]{2}{*}{ Stimulus Set } & \multicolumn{2}{|c|}{ Substitutive Components } & \multicolumn{2}{|c|}{ Additive Components } \\
\hline & $p$ & $q$ & $x$ & $y$ \\
\hline Sea Scenes & Calm & ugh & Island & Boat \\
\hline & Is & & & loud \\
\hline & rs & & & lant \\
\hline & & & wers & shtray \\
\hline & aight & Curved & Mouth & Eyebrow \\
\hline Fac & $\mathbf{H}$ & Hair & Glasses & Beard \\
\hline Scenes & Small Trees & Big Trees & Fawn & Turtle \\
\hline House & Small & Medium & Tree & Smoke \\
\hline Desk & Large & Small & Telephone & Book \\
\hline Sitting Rooms & Buffet & Chest & Picture & Lamp \\
\hline
\end{tabular}

\section{RESULTS}

Each response was classified as correct or erroneous. An error was defined as a failure to recall or as the recall of an incorrect item, including the report of a common component as distinctive or vice versa. Synonyms were accepted for both verbal and pictorial stimuli. The classification was unambiguous in all cases. For each subject we computed the percentage of correct recalls (out of 10) of common and distinctive components separately for the verbal and the pictorial stimuli, denoted $C_{v}, D_{v}, C_{p}$, and $D_{p}$, respectively. The average percentages, across all subjects, are presented in Table 3.

The data were analyzed by a four-way analysis of variance. Neither the order of presentation (first verbal or pictorial) nor the order of recall (first common or distinctive) was statistically significant $[F(1,116)=0.7$ and $F(1,116)=2.48$, respectivelyl. In contrast, feature type and modality had significant effects. Common components were recalled better than distinctive components $[F(1,116)$ $=79.92, \mathrm{MSe}=2.49, p<.001]$, probably because each common component appeared twice in the first phase, whereas each distinctive component appeared only once. Verbal components were recalled better than pictorial components $[F(1,116)=15.18, M S e=6.23, p<.001]$, but this effect is not readily interpretable because the verbal and the pictorial stimuli were not matched in salience or codability.

Our hypothesis, however, concerns the interaction between these factors. We hypothesized that the recall of common components would be enhanced by the verbal mode, whereas the recall of distinctive components would be enhanced by the pictorial mode. That is, $C_{v}+D_{p}>C_{p}$ $+D_{\nu}$. As can be seen in Table 3, the predicted interaction was confirmed $[F(1,116)=15.80, M S e=1.93$, $p<.001]$. None of the other interactions was statistically significant.

Further support for the predicted interaction emerged from the analysis of misclassified components. For each subject, we computed the percentage of common components (out of 10) reported as distinctive and the percentage of distinctive components reported as common. The average percentages, across all subjects, are presented in Table 4. 


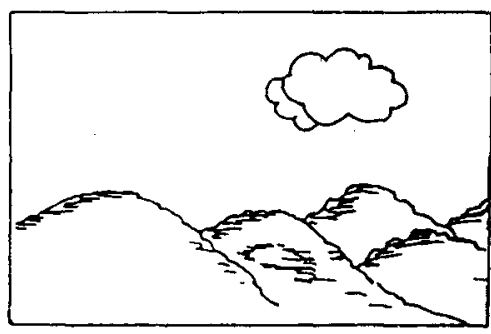

py

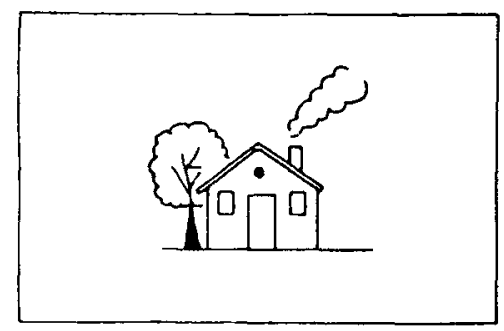

pxy

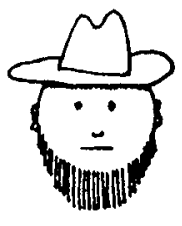

py

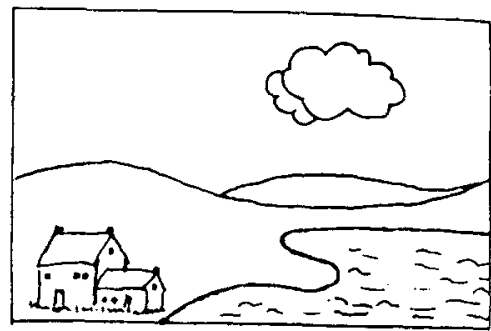

$q x y$

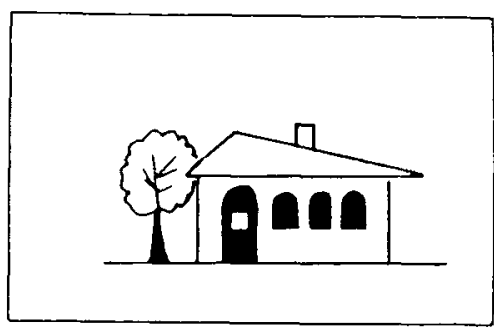

$q x$

Figure 1. Pairs of pictorial stimuli (landscapes, houses, and faces). The label below each stimulus refers to Table 2.

Overall, subjects remembered more common components they had reported as distinctive than distinctive components they had reported as common $[F(1,116)=5.80$, $M S e=0.30, p<.05]$. No significant difference between the modalities was observed $[F(1,116)=0.10, M S e=$ 0.51 . As in correct recall, there was a significant interaction between modality and feature type $[F(1,116)=$ $11.49, M S e=0.22, p<.001]$. In verbal comparisons subjects remembered more common components that they had reported as distinctive than distinctive components that they had reported as common. In pictorial comparisons, the opposite pattern was obtained.

\section{DISCUSSION}

The present experiment demonstrates that the interaction between stimulus modality and feature type, previously observed in judgments of similarity (Gati \& Tversky, 1984), is also observed in a memory task. A critical aspect of the present study is that the test of recall was preceded by similarity judgments, which facilitated the identification and the encoding of the components as common or as distinctive. The analyses of both correct recall (Table 3) and misclassified recall (Table 4) suggest that components that were encoded as common are relatively more salient in verbal than in pictorial stimuli, whereas components that were encoded as distinctive exhibit the opposite pattern. Without the prior pairing and comparison of the stimuli, their components would have been unlikely to be encoded as common or as distinctive; hence no interaction between modality and feature type would be expected.

It is very difficult to match verbal and pictorial stimuli in all relevant respects, even when they refer to the same objects. To combat this problem, we made each of the 40 verbal and pictorial components common for half of

Table 3

Percentage of Correct Recalls of Common and Distinctive Components in Verbal and Pictorial Stimuli

\begin{tabular}{lccc}
\hline & \multicolumn{3}{c}{ Stimuli } \\
\cline { 2 - 4 } Feature Type & Verbal & Pictorial & Mean \\
\hline Common & 64.0 & 50.1 & 57.0 \\
Distinctive & 46.1 & 42.2 & 44.2 \\
Mean & 55.0 & 46.2 & 50.6 \\
\hline
\end{tabular}


Table 4

Percentage of Misclassified Recalls of Common and Distinctive Components in Verbal and Pictorial Stimuli

\begin{tabular}{llll}
\hline & \multicolumn{3}{c}{ Stimuli } \\
\cline { 2 - 4 } Feature Type & Verbal & Pictorial & Mean \\
\hline $\begin{array}{l}\text { Common } \\
\text { (Reported as Distinctive) }\end{array}$ & 5.75 & 4.08 & 4.92 \\
$\begin{array}{c}\text { Distinctive } \\
\text { (Reported as Common) }\end{array}$ & 3.08 & 4.33 & 3.70 \\
Mean & 4.42 & 4.20 & 4.31 \\
\hline
\end{tabular}

the subjects and distinctive for the others. As a consequence, uncontrolled variations in the salience, the complexity, or the codability of the components could explain the difference between the verbal and the pictorial stimuli but not the interaction between modality and feature type.

Recent investigations have identified several factors that control the relative weight of common and of distinctive features (Gati \& Tversky, 1984; Tversky \& Gati, 1978). This variable is affected by (1) the nature of the task (e.g., common features loom larger in judgments of similarity than in judgments of dissimilarity), (2) baseline similarity (i.e., a common component has a greater impact when added to dissimilar than to similar stimuli), and (3) modality (i.e., common features loom larger in verbal than in pictorial comparisons). Future research may help explain the modality effect and determine whether it is reducible to other factors.

\section{REFERENCES}

GATI, I., \& TVersky, A. (1984). Weighting common and distinctive features in perceptual and conceptual judgments. Cognitive Psychology, 16, 341-370.

TVerskx, A. (1977). Features of similarity. Psychological Review, 84, 327-352.

TVERSKY, A., Gati, I. (1978). Studies of similarity. In E. Rosch \& B. B. Lloyd (Eds.), Cognition and categorization (pp. 79-98). Hillsdale, NJ: Erlbaum.

(Manuscript received July 3, 1986; revision accepted for publication July 12,1986 .) 